\title{
Correlation of surface site formation to nanoisland growth in the electrochemical roughening of $\mathrm{Pt}(111)$
}

\author{
Leon Jacobse ${ }^{\circledR}{ }^{1}$, Yi-Fan Huang', Marc T. M. Koper ${ }^{1 \star}$ and Marcel J. Rost ${ }^{2 \star}$
}

Platinum plays a central role in a variety of electrochemical devices and its practical use depends on the prevention of electrode degradation. However, understanding the underlying atomic processes under conditions of repeated oxidation and reduction inducing irreversible surface structure changes has proved challenging. Here, we examine the correlation between the evolution of the electrochemical signal of $\mathrm{Pt}(111)$ and its surface roughening by simultaneously performing cyclic voltammetry and in situ electrochemical scanning tunnelling microscopy (EC-STM). We identify a 'nucleation and early growth' regime of nanoisland formation, and a 'late growth' regime after island coalescence, which continues up to at least 170 cycles. The correlation analysis shows that each step site that is created in the 'late growth' regime contributes equally strongly to both the electrochemical and the roughness evolution. In contrast, in the 'nucleation and early growth' regime, created step sites contribute to the roughness, but not to the electrochemical signal.

E arly attempts to resolve the structure of the roughened $\mathrm{Pt}(111)$ surface were made by ex situ low-energy electron diffraction $(\mathrm{LEED})^{1,2}$, in situ X-ray reflectivity ${ }^{3-5}$, and in situ EC-STM ${ }^{6-10}$ experiments. From these studies it was concluded that the roughened surface is covered with nanoscale islands of (at most) a few atoms high. Indications were found that the resulting surface structure depends on the number of oxidation-reduction cycles (ORCs), the upper potential limit (or transferred charge), and the potential scan rate. However, it has as yet been impossible to fully resolve the island evolution. Importantly, the corresponding electrochemical data for all spatially resolved studies is either absent or incomplete.

Based on electrochemical experiments, insights into the average surface evolution were obtained during the first $20 \mathrm{ORCs}^{11-15}$. Initially both $\{100\}$ and $\{111\}$ steps (more precisely, these step sites should be denoted as $<110>/\{100\}$ and $<110>/\{111\}$ respectively, that is, steps along a direction equivalent to [110] and exhibiting a square $\{100\}$ or hexagonal $\{111\}$ geometry) form on the roughened surface, while eventually only $\{111\}$ steps remain. Advanced in situ EC-STM ${ }^{16,17}$ and X-ray reflectivity ${ }^{18-22}$ experiments are ideal tools to study this system at the atomic level. Using X-ray scattering experiments, and following the surface evolution for 20 ORCs, Ruge et al. observed an increasing island size with increasing cycle number and decreasing upper potential limit ${ }^{21}$. However, there is no single publication that provides both the detailed structural information and the complete electrochemical characterization from the very same experiment. This is a prerequisite for a true correlation, a deeper understanding of the roughening process, and the interpretation of the voltammetric signals.

Herein, we present experiments using a home-built EC-STM, in which STM images and cyclic voltammograms (CVs) were recorded alternatingly for 170 ORCs. The presented data originate from one single experiment, which was reproduced four times to ensure its representative character (for details see Methods and Supplementary Information). We first discuss the observed electrochemical signals that have been measured in situ during the EC-STM experiment and will then turn to the STM results and their analysis.

\section{Electrochemical measurements}

Figure 1 shows, from black to white, the CVs of the Pt(111) electrode of 170 ORCs between 0.06 and $1.35 \mathrm{~V}$. From the evolution of the CVs, one notices the appearance and disappearance of peaks and shoulders upon increasing cycle number. The changes in the hydrogen adsorption/desorption region $(<0.4 \mathrm{~V})$ can be related to the presence and appearance of specific step- or defect-like surface structures ${ }^{11,12,14,23-28}$. With increasing potential, first $\mathrm{OH}$ is adsorbed on the terrace $(0.8 \mathrm{~V})$, followed by the formation of a two-dimensional (2D) oxide $(1.05 \mathrm{~V})$ and a three-dimensional (3D) oxide $(>1.1 \mathrm{~V})^{15,29,30}$. Not all atoms reach their original positions upon reduction of the $3 \mathrm{D}$ oxide, leading to surface roughening. Simultaneously, a small amount of $\mathrm{Pt}^{2+}$ dissolves in the electrolyte, a part of which will be reduced on the surface once the potential is decreased ${ }^{31,32}$. The identification of specific surface sites is typically performed in the hydrogen region. Initially, this region consists only of a single broad band, associated with hydrogen adsorbed on $\mathrm{Pt}(111)$ terrace sites. The charge related to this process, indicated by the dark blue area in Fig. 1, is $168 \mu \mathrm{Ccm}^{-2}$. The agreement of this value with the literature ${ }^{33}$ and the absence of any other peaks confirm the cleanliness and efficacy of our set-up, sample treatment, and preparation.

Starting from this well-defined situation, two new peaks $\left(A_{1}\right.$ and $\left.A_{2}\right)$ appear when the sample is subjected to the first ORCs. The $A_{1}$ peak is attributed to $\{100\}$ step sites and starts decreasing again already after three cycles. In contrast, the $A_{2}$ peak, which is attributed to $\{111\}$ step sites, stabilizes after approximately 20 cycles. These observations agree well with reported data ${ }^{14}$. Interestingly, we find a third, not earlier reported, peak $\left(\mathrm{A}_{3}\right)$ that develops slowly with increasing cycle number starting around the 30th cycle. Based on other single-crystal experiments it might be related to the formation of $\{100\}$ terrace sites ${ }^{34,35}$. 


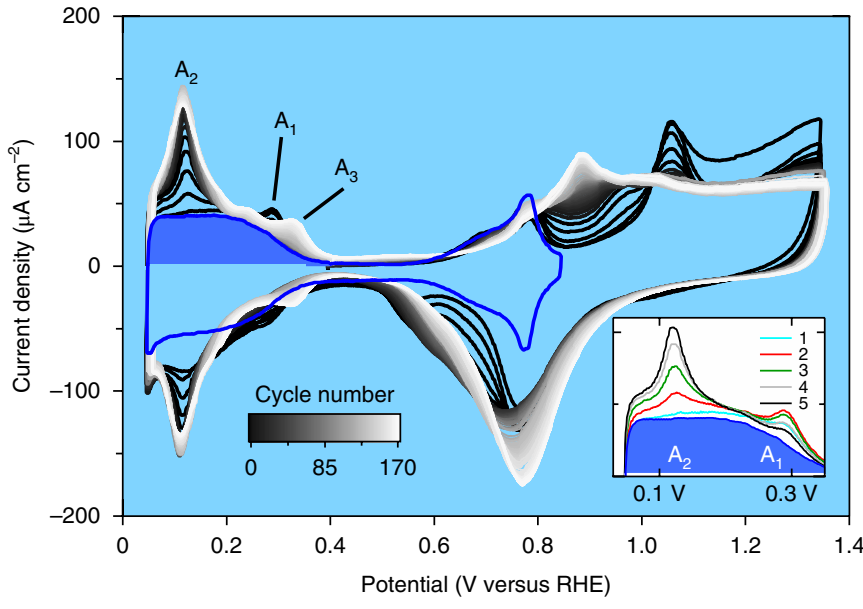

Fig. 1 | Cyclic voltammograms of consecutive oxidation-reduction cycles. The CV of the pristine $\mathrm{Pt}(111)$ surface is shown in blue and the blue shaded area indicates the initial charge related to hydrogen desorption. The scan rate is $50 \mathrm{mV} \mathrm{s}^{-1}$. The inset shows the evolution of the $A_{1}$ and $A_{2}$ peaks during the first five cycles.

The total charge related to hydrogen desorption $\left(q_{\mathrm{H}}\right)$ can be obtained by integrating the anodic, double-layer-corrected current between 0.06 and $0.4 \mathrm{~V}$ (see Supplementary Information). As $q_{\mathrm{H}}$ is directly related to the number of surface sites, it should be possible to convert it into a measure for the surface roughness. However, when converting a terrace site into the various undercoordinated (step) sites one has to take into account different stoichiometric coefficients $^{23-26}$. For fewer than 20 ORCs, there is no linear relationship between the charge corresponding to the $A_{1}$ and $A_{2}$ peaks, leading to $q_{\mathrm{H}}$ being a complex function of the created as well as annihilated adsorption sites. Further complications arise as the stoichiometric coefficients in the kinetic model describing the roughening process $^{11,12}$ differ from the ones determined for the ideally stepped single-crystal surfaces. This suggests that the formed adsorption sites should not be considered as regular step edges, although they might be so geometrically, as finite-size effects are known to change the local potential energy landscape of the sites ${ }^{36,37}$. In the following, we will use $q_{\mathrm{H}}$ to describe the electrochemically observed roughening, which we compare to the roughness derived from the EC-STM data.

\section{In situ electrochemical scanning tunnelling microscopy}

Although our STM is capable of measuring in operando during a $\mathrm{CV}$, our aim was to record high-quality snapshots, in which the complete imaged surface has transformed in the same way. Therefore, all images are acquired in the double-layer region at constant tip and sample potential $\left(U_{t}=0.45 \mathrm{~V}\right.$ and $U_{s}=0.4 \mathrm{~V}$, respectively). The full set of STM images is provided as a movie in the Supplementary Information and Fig. 2 shows four selected images illustrating the key situations in the roughening evolution. We first describe the surface changes qualitatively, before turning to a quantitative analysis.

Figure 2a shows the starting situation with large (111) terraces confirming the well-prepared and clean surface. The few islands on the terraces are remainders of our flame-annealing procedure to approximately $1,250 \mathrm{~K}$. During cooling down, the thermally activated equilibrium adatom density has to decrease, and all adatoms that can not reach a step edge within their diffusion length will nucleate as small islands $s^{38,39}$. This is confirmed by the denuded zone in front of the lower step edge, the missing denuded zone at the upper step edge (Ehrlich-Schwoebel barrier ${ }^{40,41}$ ), the distances between the islands, their size, and the fact that these islands show the standard $\mathrm{Pt}(111)$ step-height.
During the first $\sim 25$ ORCs, small, monoatomic-high adatom islands nucleate on the surface and existing islands undergo a lateral and slight height growth. This is illustrated in the STM image after eight cycles (Fig. 2b). We refer to this phase as the 'nucleation and early growth' regime of the roughening process. Simultaneous with the formation of adatom islands, vacancy islands should be created to balance the total mass. However, small vacancy islands are extremely hard to resolve with an STM, due to their convolution with the tip shape. This is probably the reason that the early EC-STM studies reported only the formation of adatom islands ${ }^{6-8}$, whereas 'holes' ${ }^{16}$ and 'pits' ${ }^{17}$ were observed only more recently. We have clear evidence for the formation of vacancy islands. First, we do observe small areas that are lower than the original pristine terrace-see the darker spots between the islands in Fig. 2b. As not all of them show a depth of exactly one step height (some half a step height), one might argue that these are oxide-like remainders from the oxidation-reduction process. This, however, is unlikely, as our holes do not change at a constant, reducing sample potential in between the ORCs (see Supplementary Information). Furthermore, the vacancy islands depth increases with increasing cycle number, which is interpreted as lateral growth, thereby reducing the effect of the tip convolution. Finally, the height line after cycle 170 in Fig. 3 clearly shows that the original terrace is etched more than two monolayers deep.

With further ORCs the nucleation of new islands stops and any further changes are related to the growth of existing islands both in lateral size and in height. The original, unaffected terrace area decreases until the islands eventually coalesce, as shown in Fig. $2 \mathrm{c}$ after 31 cycles. Tip convolution prevents the determination of the exact moment of coalescence between the 20th and 30th cycle. Depending on the precise mechanism for the island nucleation and evolution, one might expect that stagnation sets in at this point and that the surface has adapted its growth equilibrium (steady-state) structure for this process ${ }^{42,43}$. However, even up to the 170th cycle (Fig. 2d), the surface changes significantly. We describe this as the 'late growth' phase. To fully acknowledge the differences, we extracted four height lines, plotted in Fig. 3, from the STM images taken at the position indicated by the dashed line in Fig. 2d. These height lines show that initially monoatomic-high islands nucleate on the flat terrace and that the final height variation (after 170 ORCs) spans six monolayers. From the horizontal line that indicates the original terrace, it is clear that the increasing height variation is caused both by an increased island height and an increased 'depression' depth, which is due to vacancy island formation.

There have been two earlier EC-STM studies on the prolonged roughening of $\mathrm{Pt}(111)$ by ORCs, from the groups of Itaya ${ }^{8}$ and Shibata ${ }^{10}$, using a different supporting electrolyte $\left(0.5 \mathrm{M} \mathrm{H}_{2} \mathrm{SO}_{4}\right)$ and a slightly higher upper potential limit $(1.5 \mathrm{~V})$. Itaya concluded that no further surface evolution takes place after the 10th cycle while measuring up to 100 cycles. Shibata, on the other hand, reported island growth for at least 100 cycles, as determined by EC-STM. Although the surface was subjected to 1000 ORCs in the latter study, it is unclear if this led to any additional island growth. Our realspace observation, following exactly the same part of the surface in the form of STM images, enables a detailed roughness analysis which, combined with the evolution of the CVs, proves that the surface keeps on evolving up to the 170th cycle, although the changes become less pronounced with increasing cycle number.

\section{Quantitative roughness analysis}

To quantify the roughness and its evolution, we calculate from our STM images so-called height-difference correlation functions (HDCFs $)^{44}$. We use the second-order form of the family of HDCFs, because this provides direct access to the root-mean-square (RMS) roughness value. 

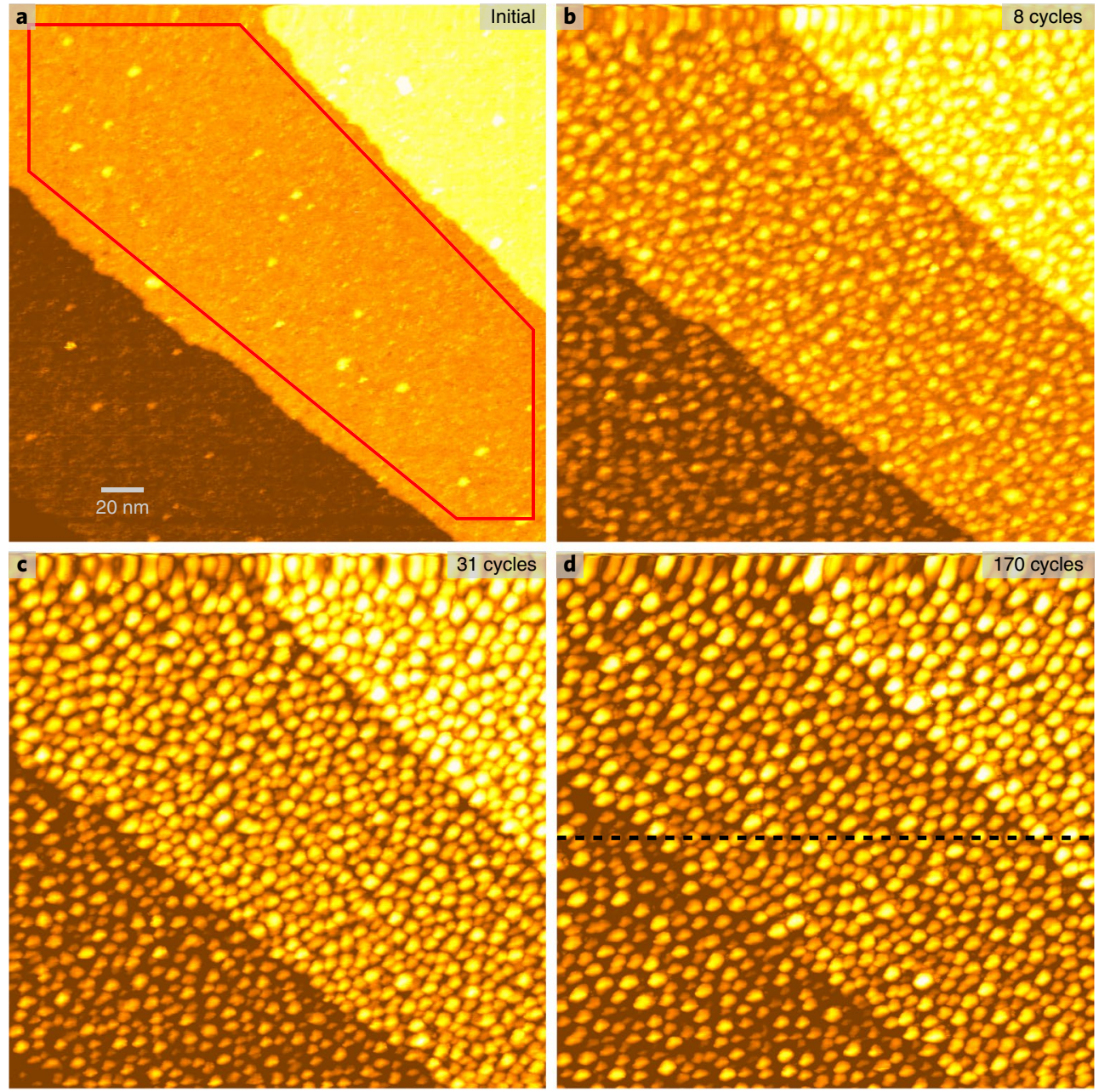

Fig. 2 | STM images after different numbers of oxidation-reduction cycles. a, Starting situation of the Pt(111) surface after cleaning. A few Pt adatom islands are visible that remain from the flame annealing (see text for more details). b. After 8 cycles, several islands, predominantly of monoatomic height, have nucleated. The original terrace is still visible in between the islands, and upon careful inspection one even sees the vacancy islands. c, After 31 cycles, the islands have grown in size such that they touch each other and cover the complete terrace. $\mathbf{d}$, After 170 cycles, the islands have continued growing, primarily in height, as is clarified in Fig. 3, which compares the height profiles of the surface at the position indicated by the dashed black line. All images $\left(230 \times 230 \mathrm{~nm}^{2}\right)$ were recorded at the same part of the surface with $U_{t}=0.45 \mathrm{~V}, U_{s}=0.4 \mathrm{~V}$, and $I_{t}<300 \mathrm{pA}$. The oxidation-reduction cycles have been performed between 0.06 and $1.35 \mathrm{~V}$ with a scan rate of $50 \mathrm{mVs}^{-1}$. The solid red line in a shows the typical area that we used for our analysis.

$$
C_{2}\left(\mathbf{x}_{0}, \mathbf{r}\right)=<\left|h\left(\mathbf{x}_{0}+\mathbf{r}\right)-h\left(\mathbf{x}_{0}\right)\right|^{2}>^{1 / 2}
$$

Specifically, we calculate the average for all different data points of the surface and for the full range of radii

$$
C_{2}(r)=\left\langle C_{2}\left(\mathbf{x}_{0}, \mathbf{r}\right)\right\rangle_{|\mathbf{r}|=r,\left|\mathbf{x}_{0}\right|}
$$

For a self-affine surface, $C_{2}(r)$ can be described with scaling parameters

$$
\begin{aligned}
& C_{2}(r)=\xi_{\perp} f\left(r / \xi_{\|}\right) \quad \text { with } \quad f(x) \sim r^{H} \quad \text { for } r \ll \xi_{\|} \\
& f(x)=\sqrt{2} \text { for } r \gg \xi_{\|}
\end{aligned}
$$

in which $\xi_{\perp}$ is the perpendicular correlation length (surface width or RMS roughness), $\xi_{\|}$is the parallel correlation length, and $H$ is the so-called Hurst exponent. The surface width describes the surface roughness, the parallel correlation length describes the lateral distance one needs to travel to reach the surface roughness, and the Hurst exponent describes how the roughness is locally built up.
Figure 4 shows $C_{2}(r)$ for all our STM images from the first to the 170th ORC (68 images in total, see Methods). To avoid contributions from the slope of the sample and the step edges, we analysed only a background-corrected part of a single terrace (see red area in Fig. 2a and Supplementary Information). For all images we observe a plateau value $\left(\sqrt{2} \xi_{\perp}\right)$ for large distances, which confirms that the chosen image size is indeed large enough to capture the full terrace roughness. At short distances, $C_{2}(r)$ shows a power-law dependence. $\xi_{\|}$is determined by calculating the intersection between $\xi_{\perp}$ and a power-law fit through the first 20 data points $(r<1.4 \mathrm{~nm})$, as illustrated by the red/white dashed lines in Fig. 4 . Initially, $\xi_{\|}$depends on both the island size and the nucleation distance. After coalescence, $\xi_{\|}$depends only on the island size. The slight increase in the 'late growth' regime indicates lateral island growth. Although $\xi_{\|}$ can not directly be translated into an absolute island size, it can be derived from the first local minimum of $C_{2}(r)$. Between 31 and 170 cycles, our average island size is $8.9 \pm 0.1 \mathrm{~nm}$. (The error is based on the variation of the position of the local minimum in the different images after radially averaging the HDCF. In the individual images, the radial variation of this minimum is about $0.9 \mathrm{~nm}$.) Our island 


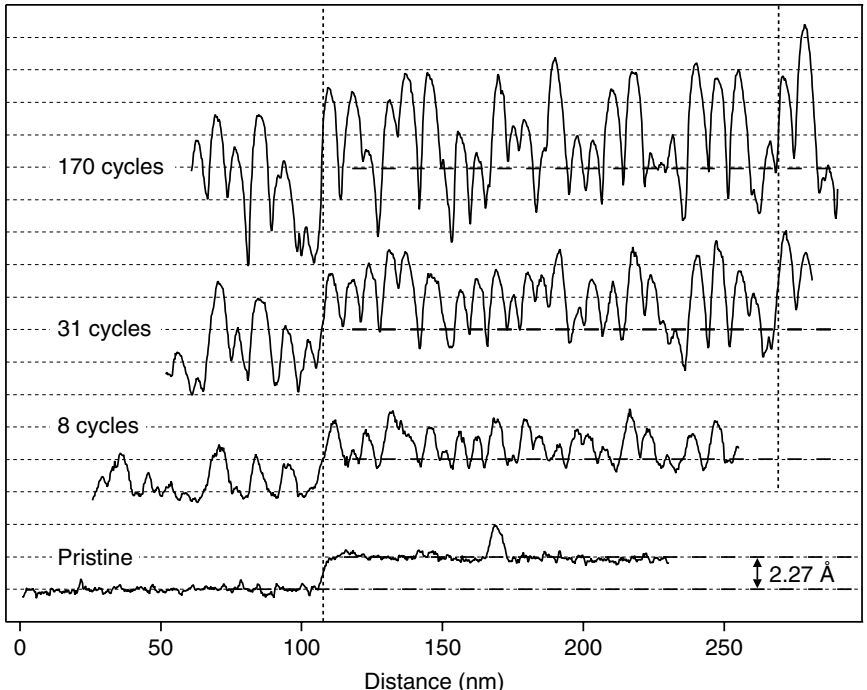

Fig. 3 | Height lines extracted from the STM images in Fig. 2. The lines are measured at the same position of the surface, allowing a direct comparison. The original step positions are indicated with the vertical dotted lines. The well-prepared, flat terrace evolves into a roughness with islands that span $6 \mathrm{MLs}$ in total. The data is vertically offset for clarity. The horizontal dashed lines indicate the original, roughened height of the upper terrace. Although the grid indicates integer step heights, one should be aware of the tipsurface convolution and, more importantly, of the low probability that a single height line captures the maximum height (depth). The fact that the original terrace is etched more than 2 MLs deep after cycle 170 proves the formation of vacancy islands.

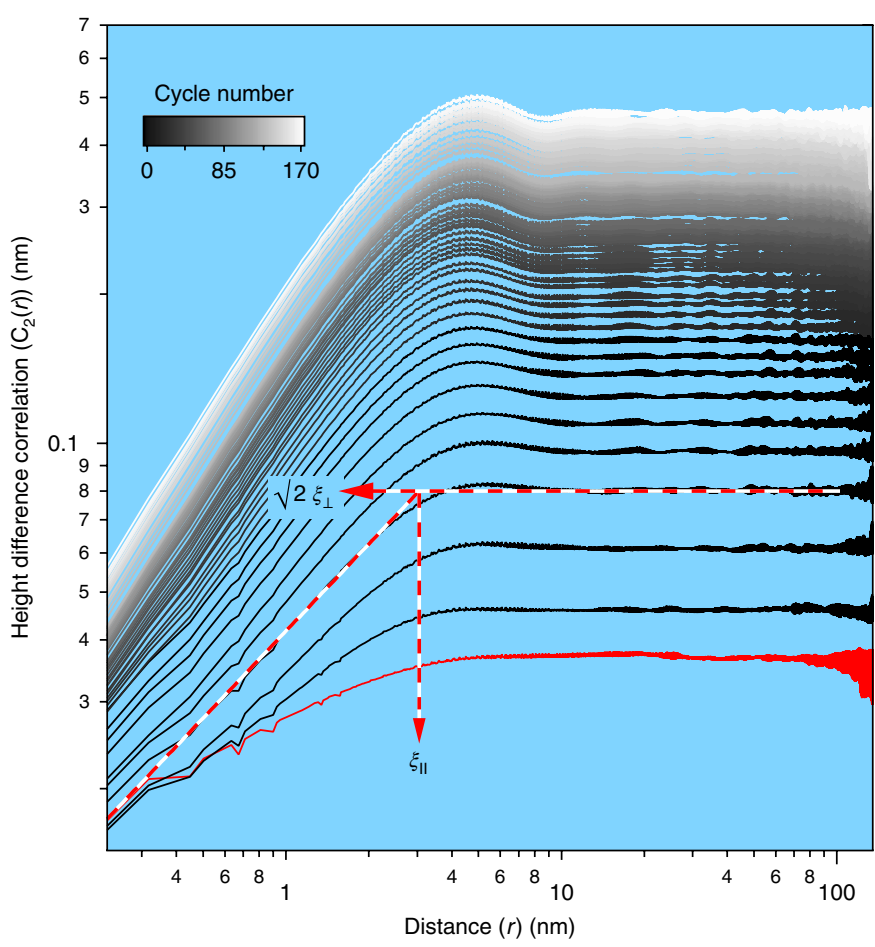

Fig. 4 | Height-difference correlation functions $\left(C_{2}(r)\right)$ as a function of ORC number. The number of the oxidation-reduction cycles is indicated in the legend and covers from black to white all 170 cycles. The result for the pristine surface is shown in red. The red/white dashed lines illustrate the determination of the surface width $\left(\xi_{\perp}\right)$ and the parallel correlation length $\left(\xi_{\|}\right)$. size is slightly larger than reported literature values ${ }^{6-10}$, which fits with the observed trend of increasing island size with decreasing upper potential limit ${ }^{21}$.

Equipped with both the electrochemical information on the number of hydrogen adsorption sites $\left(q_{\mathrm{H}}\right)$ and the detailed realspace information on the roughness $\left(\xi_{\perp}\right)$, we quantify their dependence in Fig. 5a. Figure 5b shows that the evolutions of the two curves deviates from each other until roughly the 30th cycle (that is, in the 'nucleation and early growth' phase). As mentioned before, this is also the regime where the voltammetry exhibits a complex and not yet understood behaviour. In the 'late growth' regime, however, there is a very good agreement between both curves.

To quantify the correlation between $q_{\mathrm{H}}$ and $\xi_{\perp}$, we calculate the cumulative correlation coefficient $r(n)$ shown in the inset of Fig. $5 \mathrm{~b}$ (see Methods). In the 'nucleation and early growth' regime, the value for the correlation is rather low. At the end of the 'nucleation and early growth' regime, $r(n)$ starts to increase significantly (while also becoming more accurate), eventually reaching a maximum of $r=0.87 \pm 0.02$ after 170 ORCs. Actually, omitting the initial ORCs, one would find a maximum correlation of $r(31-170)=0.96 \pm 0.01$. Thus, the transition between the two regimes occurs between the 21 st (the minimum of $r(n)$ ) and the 31st cycle. This analysis demonstrates that there is a strong and significant linear correlation between $q_{\mathrm{H}}$ and $\xi_{\perp}$ in the 'late growth' phase: it implies that the number of newly generated step edges contributing to the surface roughness is a constant fraction of the total number of newly generated hydrogen adsorption sites. The strong correlation between $q_{\mathrm{H}}$ and $\xi_{\perp}$ is in line with the qualitative observation that the islands grow predominantly in height during the 'late growth' phase.

The fact that the correlation holds over a large number of ORCs is rather surprising. On the formed nanoscale islands, one expects finitesize effects (due to stress relaxations and step-step interactions $s^{36,37,45}$ ) to change the atomic distances and the local potential energy landscape, leading to a more complicated evolution of the electrochemical signals. The derivation of an atomic model that describes the island shapes during the evolution based on the EC-STM data should lead to a deeper insight into (the evolution of) the voltammetric features.

More detailed information on the roughening mechanism can be obtained by further analyzing the time evolution of $\xi_{\perp}$ and $\xi_{\|}$. If the underlying mechanism were to remain the same during the entire experiment and the continuous process can be described by one set of differential equations, so-called scaling should occur and the time evolution should adhere to the following three power-law dependencies:

$$
\begin{aligned}
& \xi_{\perp} \sim t^{\beta} \\
& \xi_{\|} \sim t^{1 / z} \sim t^{\beta / \alpha} \\
& \xi_{\perp} \sim \xi_{\|}^{\alpha}
\end{aligned}
$$

The values of the scaling exponents $(\alpha, \beta, z)$ allow one to classify data according to specific universality classes ${ }^{44}$. The different classes describe, for example, the influences of diffusion via the surface or the electrolyte, mass conservation, or etching/deposition flux dependence on the surface dynamics ${ }^{44}$. Strictly speaking, scaling is applicable only to a continuous evolution in time. However, it was demonstrated that this formalism holds also for our kind of electrochemical experiments, with interrupted growth and surface evolution, as long as all surface changes occur only during the oxidation/ reduction transition (negligible surface diffusion in between) ${ }^{3}$.

If ideal scaling were to occur, the logarithmic representation of $\xi_{\perp}$ versus cycle number in Fig. 5b should yield a single straight line. However, we observe at least two slopes, and therefore scaling does not apply over all 170 cycles. Considering the two growth regimes 

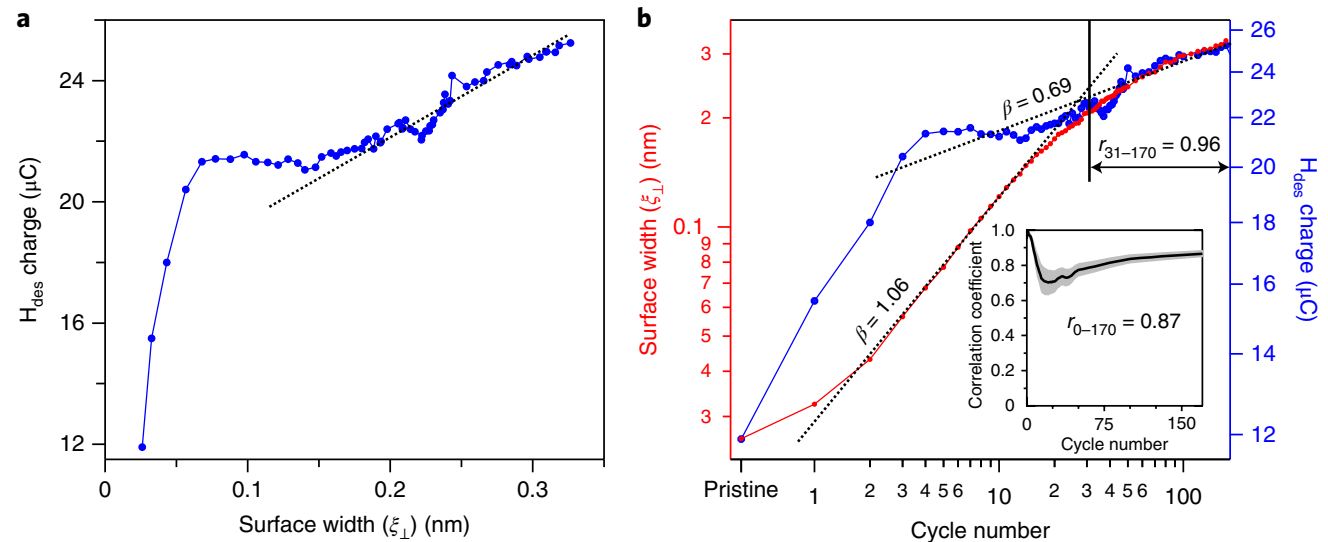

Fig. 5 | Correlation between electrochemical and STM data. a, Hydrogen desorption charge as a function of the surface width. The dotted black line, indicating the linear correlation in the 'late growth' regime, is drawn to guide the eye. $\mathbf{b}$, The surface width (red, from STM images) is shown together with the charge related to hydrogen desorption (blue, from cyclic voltammetry). Both sets of data are plotted logarithmically to easily identify possible power-law regimes. The $y$-axes are chosen such that the data for the initial and final situation overlap. The two black dotted lines indicate the two possible scaling regimes as discussed in the main text. Details on the determination of the exponent $\beta$ are provided in the Supplementary Information. The inset shows the cumulative correlation coefficient quantifying the strong correlation between the STM and electrochemical data. The shaded area indicates its probable error ${ }^{48}$.

already identified, this is expected. As nucleation may also follow scaling laws ${ }^{46}$, the roughness in Fig. 5 might be described by two sets of exponents for the different regimes, indicated by the two black dotted lines.

Previously, You and Nagy concluded from X-ray reflectivity experiments on the same system that scaling does occur during the first nine ORCs. Slight differences in the roughening process might occur as they kept the surface at $1.25 \mathrm{~V}$ for 15 minutes, whereas we scan the sample potential to $1.35 \mathrm{~V}$. However, You and Nagy discussed only the first of the three power laws in equation (4), which can not indisputably prove scaling. In our case, the nonlinear behaviour of $\xi_{\|}$, with a pronounced maximum during the first nine ORCs (see Supplementary Information), leads to the conclusion that scaling does not occur in this regime.

The 'late growth' regime can be described by the power laws in equation (4), but also this analysis does not lead to consistent values for the exponents to prove scaling (see Supplementary Information). This is a well-known issue, as the 'ideal' values for the scaling exponents are approached asymptotically when the growth mechanism changes from one process to another ${ }^{44}$. Therefore, we can also not say that scaling does not occur in the 'late growth' regime. An even larger number of ORCs would be necessary to provide more insight.

If scaling does not occur and the growth is conserved, the surface profile might end in a steady-state situation in the 'late growth' regime $^{42,43}$. However, a small fraction of a monolayer of platinum dissolves during each $\mathrm{ORC}^{31,32}$. Moreover, the amount of Pt that dissolves during each ORC increases with increasing cycle number for at least $100 \mathrm{ORCs}^{47}$. Although it would be possible to reach a steadystate surface structure while losing material into the electrolyte during each cycle ${ }^{42,43}$, the fact that the dissolved amount changes with each cycle means that the surface can not reach such a steady state.

\section{Conclusions}

In this work, we have for the first time directly linked electrochemical observations to structural information obtained from high-resolution, real-space EC-STM images of $\mathrm{Pt}(111)$ subjected to oxidation-reduction cycles. This approach allows for a quantitative correlation between electrochemical and STM data. Our experiments show that the total roughening process can be divided into two regimes: 'nucleation and early growth', followed by 'late growth'. During the 'nucleation and early growth' phase the electrode surface is covered with an increasing number of nanoscale islands.
Simultaneously, these islands also increase in radius and slightly in height. In this regime, the roughness extracted from the STM images is not linearly correlated to the hydrogen desorption charge determined from cyclic voltammetry, as geometrically created step sites do not contribute to the electrochemical signal. The analysis to identify the underlying mechanism during the 'nucleation and early growth' phase indicates that no scaling occurs. Once the islands have coalesced, the existing islands keep growing, predominantly in height. In this 'late growth' phase, there is a linear correlation between the surface roughness and the hydrogen desorption charge. In this regime, in contrast to the 'nucleation and early growth' regime, each created step site contributes equally to the electrochemical signal as well as to the roughness. Future research is necessary to understand the lack of correlation in the 'nucleation and early growth' regime. Our experiment consisting of 170 ORCs is not extensive enough to completely (dis)prove scaling during the 'late growth' phase. A key factor in the long-term evolution of the roughened $\mathrm{Pt}(111)$ surface is probably the increasing amount of dissolving Pt with prolonged potential cycling.

\section{Methods}

Methods, including statements of data availability and any associated accession codes and references, are available at https://doi. org/10.1038/s41563-017-0015-z.

Received: 8 August 2017; Accepted: 21 December 2017; Published online: 12 February 2018

\section{References}

1. Wagner, F. T. \& Ross, P. N. LEED spot profile analysis of the structure of electrochemically treated $\mathrm{Pt}(100)$ and $\mathrm{Pt}(111)$ surfaces. Surf. Sci. 160, 305-330 (1985).

2. Aberdam, D., Durand, R., Faure, R. \& El-Omar, F. Structural changes of a $\mathrm{Pt}(\mathrm{lll})$ electrode induced by electrosorption of oxygen in acidic solutions: a coupled voltammetry, LEED and AES study. Surf. Sci. 171, 303-330 (1986).

3. You, H. \& Nagy, Z. Oxidation-reduction-induced roughening of platinum (111) surface. Physica B 198, 187-194 (1994).

4. You, H., Zurawski, D. J., Nagy, Z. \& Yonco, R. M. In-situ X-ray reflectivity study of incipient oxidation of $\mathrm{Pt}(111)$ surface in electrolyte solutions. J. Chem. Phys. 100, 4699-4702 (1994).

5. Nagy, Z. \& You, H. Applications of surface X-ray scattering to electrochemistry problems. Electrochim. Acta 47, 3037-3055 (2002).

6. Sugawara, S. \& Itaya, K. In situ scanning tunnelling microscopy of a platinum $\{111\}$ surface in aqueous sulphuric acid solution. J. Chem. Soc., Faraday Trans. 1 85, 1351-1356 (1989). 
7. Itaya, K. In situ scanning tunneling microscopy of platinum (111) surface with the observation of monatomic steps. J. Vac. Sci. Technol. A 8 , 515-519 (1990).

8. Sashikata, K., Furuya, N. \& Itaya, K. In situ electrochemical scanning tunneling microscopy of single-crystal surfaces of $\mathrm{Pt}(111), \mathrm{Rh}(111)$, and $\mathrm{Pd}(111)$ in aqueous sulfuric acid solution. J. Vac. Sci. Technol. B 9, 457-564 (1991).

9. Breuer, N., Funtikov, A., Stimming, U. \& Vogel, R. In situ electrochemical STM imaging of roughened gold and platinum electrode surfaces. Surf. Sci. 335, 145-154 (1995)

10. Furuya, N. \& Shibata, M. Structural changes at various Pt single crystal surfaces with potential cycles in acidic and alkaline solutions. J. Electroanal. Chem. 467, 85-91 (1999).

11. Björling, A., Ahlberg, E. \& Feliu, J. M. Kinetics of surface modification induced by submonolayer electrochemical oxygen adsorption on $\operatorname{Pt}\left(\begin{array}{lll}1 & 1 & 1\end{array}\right)$. Electrochem. Commun. 12, 359-361 (2010).

12. Björling, A. \& Feliu, J. M. Electrochemical surface reordering of $\mathrm{Pt}(111)$ : A quantification of the place-exchange process. J. Electroanal. Chem. 662, 17-24 (2011).

13. Björling, A., Herrero, E. \& Feliu, J. M. Electrochemical oxidation of Pt $\left(\begin{array}{lll}1 & 1 & 1\end{array}\right)$ vicinal surfaces: effects of surface structure and specific anion adsorption. J. Phys. Chem. C 115, 15509-15515 (2011).

14. Gómez-Marín, A. M. \& Feliu, J. M. Pt(111) surface disorder kinetics in perchloric acid solutions and the influence of specific anion adsorption. Electrochim. Acta 82, 558-569 (2012).

15. Gómez-Marín, A. M. \& Feliu, J. M. Oxide growth dynamics at Pt(111) in absence of specific adsorption: A mechanistic study. Electrochim. Acta 104, 367-377 (2013).

16. Löffler, T., Bussar, R., Xiao, X., Ernst, S. \& Baltruschat, H. The adsorption of ethene on vicinally stepped electrode surfaces and the effect of temperature. J. Electroanal. Chem. 629, 1-14 (2009).

17. Wakisaka, M., Asizawa, S., Uchida, H. \& Watanabe, M. In situ STM observation of morphological changes of the $\mathrm{Pt}(111)$ electrode surface during potential cycling in $10 \mathrm{mM}$ HF solution. Phys. Chem. Chem. Phys. 12, 4184-4190 (2010).

18. Liu, Y., Barbour, A., Komanicky, V. \& You, H. X-ray crystal truncation rod studies of surface oxidation and reduction on $\mathrm{Pt}(111)$. J. Phys. Chem. C. 120, 16174-16178 (2016).

19. Goryachev, A. et al. Synchrotron based operando surface X-ray scattering study towards structure-activity relationships of model electrocatalysts. ChemistrySelect 1, 1104-1108 (2016).

20. Drnec, J. et al. Initial stages of $\mathrm{Pt}(111)$ electrooxidation: dynamic and structural studies by surface X-ray diffraction. Electrochim. Acta 224, 220-227 (2017).

21. Ruge, M. et al. Structural reorganisation of $\mathrm{Pt}(111)$ electrodes by electrochemical oxidation and reduction. J. Am. Chem. Soc. 139, 4532-4539 (2017).

22. Ruge, M. et al. Electrochemical oxidation of smooth and nanoscale rough Pt(111): An in situ surface X-ray scattering study. J. Electrochem. Soc. 164 H608-H614 (2017).

23. Clavilier, J., El Achi, K. \& Rodes, A. In situ characterization of the Pt(S)$[\mathrm{n}(111) \times(111)]$ electrode surfaces using electrosorbed hydrogen for probing terrace an step sites. J. Electroanal. Chem. 272, 253-261 (1989).

24. Rodes, A., El Achi, K., Zamakhchari, M. A. \& Clavilier, J. Hydrogen probing of step and terrace sites on $\mathrm{Pt}(\mathrm{S})-[\mathrm{n}(111) \times(100)]$ electrodes. J. Electroanal. Chem. 284, 245-253 (1990).

25. Rodes, A. \& Clavilier, J. Electrochemical study of step reconstruction on platinum surfaces belonging to the [011] zone between $\mathrm{Pt}(311)$ and $\mathrm{Pt}(111)$. J. Electroanal. Chem. 344, 269-288 (1993).

26. Clavilier, J. \& Rodes, A. Electrochemical detection and characterization at $\mathrm{Pt}(\mathrm{N}, \mathrm{N}, \mathrm{N}-2)$ oriented electrodes of multiatomic step formation induced by quenching at high-temperatures. J. Electroanal. Chem. 348, 247-264 (1993).

27. Solla-Gullón, J., Rodríguez, P., Herrero, E., Aldaz, A. \& Feliu, J. M. Surface characterization of platinum electrodes. Phys. Chem. Chem. Phys. 10, 1359-1373 (2008).

28. Vidal-Iglesias, F. J., Arán-Ais, R. M., Solla-Gullón, J., Herrero, E. \& Feliu, J. M. Electrochemical characterization of shape-controlled Pt nanoparticles in different supporting electrolytes. ACS Catal. 2, 901-910 (2012).

29. Van Der Niet, M. J. T. C., Garcia-Araez, N., Hernández, J., Feliu, J. M. \& Koper, M. T. M. Water dissociation on well-defined platinum surfaces: The electrochemical perspective. Catal. Today 202, 105-113 (2013).
30. Huang, Y.-F., Kooyman, P. J. \& Koper, M. T. M. Intermediate stages of electrochemical oxidation of single-crystalline platinum revealed by in situ Raman spectroscopy. Nat. Commun. 7, 12440 (2016).

31. Topalov, A. A. et al. Dissolution of platinum: Limits for the deployment of electrochemical energy conversion? Angew. Chem. Int. Ed. 51, 12613-12615 (2012).

32. Lopes, P. P. et al. Relationships between atomic level surface structure and stability/activity of platinum surface atoms in aqueous environments. ACS Catal. 6, 2536-2544 (2016).

33. Gomez, R., Orts, J. M., Alvarez-Ruiz, B. \& Feliu, J. M. Effect of temperature on hydrogen adsorption on $\mathrm{Pt}(111), \mathrm{Pt}(110)$, and $\mathrm{Pt}(100)$ electrodes in $0.1 \mathrm{M}$ $\mathrm{HClO}_{4}$. J. Phys. Chem. B 108, 228-238 (2004).

34. Furuya, N. \& Koide, S. Hydrogen adsorption on platinum single-crystal surfaces. Surf. Sci. 220, 18-28 (1989).

35. Domke, K., Herrero, E., Rodes, A. \& Feliu, J. M. Determination of the potentials of zero total charge of $\mathrm{Pt}(100)$ stepped surfaces in the [011] zone. Effect of the step density and anion adsorption. J. Electroanal. Chem. 552, 115-128 (2003).

36. Giesen, M. \& Schulze Icking-Konert, G. \& Ibach, H. Interlayer mass transport and quantum confinement of electronic states. Phys. Rev. Lett. 82, 3101-3104 (1999).

37. Lee, J., Lee, J., Tanaka, T. \& Mori, H. In situ atomic-scale observation of melting point suppression in nanometer-sized gold particles. Nanotechnology 20, 475706 (2009)

38. Michely, T. \& Krug, J. Islands, Mounds and Atoms (Springer, Berlin, Heidelberg, 2004).

39. Ibach, H. Physics of Surfaces and Interfaces (Springer, Berlin, Heidelberg, 2006).

42. Sekerka, R. F. in Crystal Growth - From Fundamentals to Technology 1st edn (eds Georg Müller, J.-J. M. \& Rudolph, P.) 55-93 (Elsevier, Amsterdam, 2004).

43. Sekerka, R. F. Equilibrium and growth shapes of crystals: How do they differ and why should we care? Cryst. Res. Technol. 40, 291-306 (2005).

44. Meakin, P. Fractals, Scaling and Growth Far from Equilibrium 119-159 (Cambridge Univ. Press, Cambridge, 1998).

45. Giesen, M. \& Ibach, H. On the mechanism of rapid mound decay. Surf. Sci. 464, L697-L702 (2000).

46. Tong, W. M. \& Williams, R. S. Kinetics of surface growth: Phenomenology, scaling, and mechanisms of smoothening and roughening. Annu. Rev. Phys. Chem. 45, 401-438 (1994).

47. Sugawara, Y., Sasaki, M., Muto, I. \& Hara, N. Dissolution of platinum single crystal surfaces under potential cycling in sulfuric acid solution. ECS Trans. 64, 81-87 (2014).

51. Güell, A. G., Díez-Pérez, I., Gorostiza, P. \& Sanz, F. Preparation of reliable probes for electrochemical tunneling spectroscopy. Anal. Chem. 76, 5218-5222 (2004)

\section{Acknowledgements}

The authors acknowledge LVH Coatings for supplying their Clearclad electrophoretic paint. This work is financially supported by the European Commission Horizon 2020 - Research and Innovation Framework Programme (Marie Skłodowska-Curie Actions Individual Fellowship awarded to Y.-F.H., No. 661145, DYNECAT).

\section{Author Contributions}

All authors contributed to the design of the experiment and the interpretation of the data. L.J., Y.-F.H. and M.J.R. performed the experimental work. L.J. performed the data analysis and manuscript preparation with the input of M.J.R. and M.T.M.K.

\section{Competing interests}

The authors declare no competing financial interests.

\section{Additional information}

Supplementary information accompanies this paper at https://doi.org/10.1038/s41563017-0015-z.

Reprints and permissions information is available at www.nature.com/reprints.

Correspondence and requests for materials should be addressed to M.T.M.K. or M.J.R. Publisher's note: Springer Nature remains neutral with regard to jurisdictional claims in published maps and institutional affiliations. 


\section{Methods}

All experiments were carried out with the home-built EC-STM set-up described previously $y^{49,50}$. This set-up has been shown to deliver high-resolution images, also on relatively rough surfaces, while still operating with significant scanning speeds. To allow for long-term experiments, for maintaining accurate control over the potentials, and for a high degree of cleanliness, we developed a new EC-STM cell with a reversible hydrogen reference electrode (RHE) that is compatible with the STM. A coiled platinum wire is used as counter electrode.

STM tips are electrochemically etched from $\mathrm{Pt}_{80} \mathrm{Ir}_{20}$ wire (Goodfellow) and coated with electrophoretic paint (Clearclad HSR) and polyethylene to minimize faradaic contributions to the tunnelling current $\left(I_{t}\right)^{51}$. The $\operatorname{Pt}(111)$ sample (cut and polished $<0.1^{\circ}$, Surface Preparation Laboratory, Zaandam, The Netherlands) is prepared by repeated cycles of mild etching $( \pm 2 \mathrm{~V}$ versus $\mathrm{Pt}$, for $2.5 \mathrm{~s}$ at $50 \mathrm{~Hz}$ in an acidified $\mathrm{CaCl}_{2}$ solution), flame annealing ( $5 \mathrm{~min}$ at $\left.\sim 1,250 \mathrm{~K}\right)$, and cooling in a reducing atmosphere (1:4 $\mathrm{H}_{2} / \mathrm{Ar}$ mixture). After the last cooling step, the surface is protected with ultrapure water $(>18.2 \mathrm{M} \Omega \mathrm{cm}$, Millipore Milli-Q) saturated with $\mathrm{H}_{2} / \mathrm{Ar}$. After each experiment the sample is sonicated in acetone to remove the black residue left by the Viton O-seal.

All parts of the electrochemical flow cell and the electrolyte reservoir are cleaned in freshly prepared piranha (3:1 $\mathrm{v} / \mathrm{v} \mathrm{H}_{2} \mathrm{SO}_{4}$ (Sigma-Aldrich, Puriss. p.a.) and $\mathrm{H}_{2} \mathrm{O}_{2}$ (Merck)) followed by at least five times boiling in ultrapure water. The tubing through which the electrolyte flows is cleaned with a diluted piranha solution and extensively rinsed with ultrapure water. The $0.1 \mathrm{M} \mathrm{HClO}_{4}$ electrolyte is prepared from high-purity perchloric acid (Merck Suprapur) and purged with $\mathrm{N}_{2}$ for at least $2 \mathrm{~h}$ prior to the experiment.

Before starting the experiment, the surface quality and cleanliness were checked by (large scale) STM imaging and cyclic voltammetry. Under these conditions the sample potential $\left(U_{s}\right)$ is not scanned above $0.85 \mathrm{~V}$ versus RHE to prevent any change in the surface structure. Directly before and after each oxidation-reduction cycle, we again record CVs up to $0.85 \mathrm{~V}$ to monitor the appearance of possible contaminants in between the oxidation experiments. All CVs are performed with the tip fully retracted $(\sim 1 \mu \mathrm{m}$ away $)$ and at a fixed potential $\left(U_{\mathrm{t}}=0.45 \mathrm{~V}\right)$ to minimize the number of tip switches. Performing the ORCs with a retracted tip also prevents changes in the local sample potential due to the extremely small distance and large potential difference between the two electrodes. Such local potential changes would imply that the imaged area is no longer representative for the entire electrode surface. After each oxidationreduction sequence (single ORCs for cycle 1-50, five ORCs for cycle 55-100, and ten ORCs for cycle 110-170) an STM image is acquired. During imaging the sample and tip potentials are fixed at $U_{s}=0.4 \mathrm{~V}$ and $U_{t}=0.45 \mathrm{~V}$, respectively. This approach leads to a total data acquisition time of $\sim 17 \mathrm{~h}$.
We define the cumulative correlation coefficient, quantifying the correlation between the hydrogen desorption charge and the surface width, as:

$$
r(n)=\frac{\sum_{i=0}^{n}\left(q_{\mathrm{H}, i}-q_{\mathrm{H},(0-n)}\right)\left(\xi_{\perp, i}-\xi_{\perp,(0-n)}\right)}{\sqrt{\sum_{i=0}^{n}\left(q_{\mathrm{H}, i}-q_{\mathrm{H},(0-n)}\right)^{2}} \sqrt{\sum_{i=0}^{n}\left(\xi_{\perp, i}-\xi_{\perp,(0-n)}\right)^{2}}}
$$

in which $n$ is the cycle number. $q_{\mathrm{H}}$ and $\xi_{\text {(n) }}$ are the mean hydrogen desorption charge and the mean surface width of the appropriate interval, respectively. The validity of $r(n)$ is determined by calculating its probable error as defined in the literature: ${ }^{48}$

$$
\text { probable error }=\frac{0.6745\left(1-r^{2}\right)}{\sqrt{N}}
$$

where $N$ is the number of data points taken into account.

Data availability. The original data sets generated and analysed during the current study are not publicly available, as they are obtained with home-written control software that is not publicly available. No other existing software is capable of reading our original microscopy data. However, an export into a standard format could be generated by the corresponding author upon reasonable request. In addition, all uncompressed microscopy images that support the findings of this study can be downloaded in form of a movie via the Supplementary Information. All data processing is performed with home-written routines using WaveMetrics Igor Pro software. Details are provided in the Supplementary Information.

\section{References}

40. Ehrlich, G. \& Hudda, F. G. Atomic view of surface self-diffusion: tungsten on tungsten. J. Chem. Phys. 44, 1039-1049 (1966).

41. Schwoebel, R. L. \& Shipsey, E. J. Step motion on crystal surfaces. J. Appl. Phys. 37, 3682-3686 (1966).

48. Eells, W. C. Formulas for probable errors of coefficients of correlation. J. Am. Stat. Assoc. 24, 170-173 (1929).

49. Yanson, Y. I. \& Rost, M. J. Structural accelerating effect of chloride on copper electrodeposition. Angew. Chem. Int. Ed. 52, 2454-2458 (2013)

50 Yanson, Y. I., Schenkel, F. \& Rost, M. J. Design of a high-speed electrochemical scanning tunneling microscope. Rev. Sci. Instrum. 84, 023702 (2013). 